\title{
On uniqueness and stability for a thermoelastic theory
}

\author{
R Quintanilla * \\ Departamento de Matemáticas, UPC \\ C.Colón 11, 08222 Terrassa, \\ Barcelona, Spain
}

June 6, 2017

\begin{abstract}
In this paper we investigate a thermoelastic theory obtained from the Taylor approximation for the heat flux vector proposed by Choudhuri. This new thermoelastic theory gives rise to interesting mathematical questions. We here prove an uniqueness theorem and instability of solutions under the relaxed assumption that the elasticity tensor can be negative. Later we consider the one dimensional and homogeneous case and we prove the existence of solutions. We finish the paper by proving the slow decay of the solutions. That means that the solutions do not decay in a uniform exponential way. This last result is relevant if it is compared with other thermoelastic theories where the decay of solutions for the one dimensional case is of exponential way.
\end{abstract}

Keywords: Thermoelastodynamics; Uniqueness; Instability; Existence; Slow decay.

\section{Introduction}

Fourier's law implies the instantaneous propagation of the heat waves. This fact is not well accepted from the thermomechanical point of view because it contradicts the causality principle. Therefore, a big interest has been developed to propose alternative constitutive equations to the Fourier law. We recall the classical formulations of Lord-Shulman [9] and Green-Lindsay [4] which are based on the Cattaneo-Maxwell heat conduction. In the last decade of the 20th century two new theories were proposed. On the one side, Green and Naghdi $[5,6]$ developed a thermomechanical theory of deformable continua that relies

* Supported by the Project "Anáisis Matemático de las Ecuaciones en Derivadas Parciales de la Termomecánica "(MTM2013-42004-P) of the Spanish Ministry of Economy and Competitiveness. 
on an entropy balance law rather than on an entropy inequality. Depending on the constitutive variables Green and Naghdi considered three cases that they denominated as types I, II and III respectively. The linear version of the type I recovers the Fourier theory. The linearized form of the type II theory does not sustain energy dissipation and permits the transmission of heat as thermal waves at finite speed. Type III is the most general and contents type I and type II as particular cases. On the other side, in 1995 Tzou [24] proposed another theory where the Fourier law is substituted by

$$
q\left(x, t+\tau_{q}\right)=-k \nabla \theta\left(x, t+\tau_{\theta}\right), \quad k>0 .
$$

Here $q$ is the heat flux vector, $\theta$ is the temperature and $\tau_{q}$ and $\tau_{\theta}$ are the delay parameters which are assumed positive. This equation says that the temperature gradient established across a material volume at the position $x$ at time $t+\tau_{\theta}$ results in a heat flux to flow at a different instant of time $t+\tau_{q}$. The delays are understood in terms of the microstructure of the material. An extension was proposed by Choudhuri [2]. In this theory the constitutive equation is

$$
q\left(x, t+\tau_{q}\right)=-\left(k \nabla \theta\left(x, t+\tau_{\theta}\right)+\kappa \nabla \nu\left(x, t+\tau_{\nu}\right)\right) .
$$

Here $\nu$ is the thermal displacement that satisfies $\nu_{t}=\theta, \kappa$ is a new parameter which is typical in the type II and III thermoelastic theories proposed by Green and Naghdi, and $\tau_{\nu}$ is another delay parameter which is also assumed positive. One thinks that the intention of Choudhuri was to propose a theory with delays such that when the Taylor approximations are considered, the theories of Green and Nagdhi are recovered.

Equations (1.1) and (1.2) are strongly based on an intuitive point of view, but there is no a priori thermomechanical foundation. Furthermore, it can be proved that, when we combine the proposed constitutive equations with the classical energy equation

$$
-\operatorname{div} q(x, t)=c \theta_{t}(x, t), \quad c>0,
$$

we can find a sequence of solutions of the form

$$
\theta_{n}(x, t)=\exp \left(\omega_{n} t\right) \Phi_{n}(x)
$$

where the real part of $\omega_{n}$ tends to infinity [3]. Therefore continuous dependence on initial data cannot be obtained, and the associated mathematical problem is ill posed in the sense of Hadamard. This fact disagrees with the a priori expectation. For this reason many people have wanted to study different formal Taylor approximations to the the delay equations [1, 12, 13, 20, 21, 22]. These alternative theories allow to obtain stability of solutions and the well-posedness of the problems, provided that certain conditions on the parameters hold.

In recent papers Quintanilla $[16,17,18]$ has proposed several modifications of the theories of Tzou and Choudhuri to obtain heat conduction equations with delay. In the last reference it was proposed to consider the limit case when $\tau_{q}=\tau_{\theta}$ and $\tau=\tau_{\nu}-\tau_{q}$. That is

$$
q(x, t)=-(k \nabla \theta(x, t)+\kappa \nabla \nu(x, t+\tau)) .
$$


And it was shown that, in this case, a result of continuous dependence on the initial data and supply terms can be obtained. In that work it was also pointed out the possibility to substitute the delay $\tau_{\nu}-\tau_{q}$ by the second order Taylor approximation to obtain the constitutive equation

$$
q(x, t)=-k \nabla \theta(x, t)-\kappa\left(\nabla \nu(x, t)+\tau \nabla \dot{\nu}+\frac{\tau^{2}}{2} \nabla \ddot{\nu}\right) .
$$

This new heat conduction equation also proposes new and stimulating mathematical questions. It is worth citing the reference [7] where the spatial behavior of the solutions of the equation proposed by the constitutive equation (1.5) was studied. This constitutive equation can be combined with the elasticity equation to obtain a new thermoelastic problem. The aim of this contribution is the study of this new thermoelastic problem. It is worth noting that in a recent paper Kumari and Mukhopadhayay [10] considered this coupled theory and proved some basic theorems. One of the results were the uniqueness of solutions using the standard energy arguments. In the present paper we will prove the uniqueness and instability of solutions when the elasticity tensor is not definite and the existence and slow decay of solutions for the one-dimensional case. To be precise, we first use the logarithmic convexity argument to obtain the uniqueness and the instability of solutions. As we do not assume the positiviness of the elasticity tensor our uniqueness result is new and it applies to a larger class of problems than the ones analysed at [10]. The logarithmic convexisty argument is a powerful tool to study the qualitative properties of the solutions of several thermoelastic problems $[11,14]$. We also use the semigroup arguments to prove the existence of solutions. We restrict our study to the one dimensional case to be coherent with the last section where we prove the slow decay of solutions for the one dimensional problem. This result is relevant if it is compared with the ones obtained in other thermoelastic theories (see $[8,15,19,23]$ among others), because this is the first case of a thermoelastic theory where the decay of solutions in the one dimensional case is not exponential.

\section{Preliminaries}

We consider a body that at time $t_{0}$ occupies the properly regular region $B$ of the Euclidean three-dimensional space and is bounded by the surface $\partial B$. The motion of the body is referred to a fixed system of rectangular cartesian axes $O x_{i}(i=1 ; 2 ; 3)$. We denote by $n_{k}$ the outward unit normal of $B$. We shall employ the usual summation and differentiation conventions: Latin subscripts, unless otherwise specified, are understood to range over the integers $(1,2,3)$, summation over repeated subscripts is implied, and subscripts preceded by a comma denote partial differentiation with respect to the corresponding cartesian coordinate. We use a superposed dot to denote partial differentiation with respect to the time.

The basic evolution equations are the equations of motion:

$$
\sigma_{i j, j}+f_{i}=\rho \ddot{u}_{i},
$$


and the equation of the energy

$$
T_{0} \dot{\eta}=-q_{i, i}+h .
$$

The constitutive equations are

$$
\begin{gathered}
\sigma_{i j}=C_{i j k l} u_{k, l}-\beta_{i j} \theta, \\
\eta=c \theta+\beta_{i j} u_{i, j},
\end{gathered}
$$

and

$$
q_{i}=-\kappa_{i j} \nu_{, j}-\left(k_{i j}+\tau \kappa_{i j}\right) \theta_{, j}+\frac{\tau^{2}}{2} \kappa_{i j} \ddot{\nu}_{, j} .
$$

Here $u_{i}$ is the displacement, $\sigma_{i j}$ is the stress tensor, $f_{i}$ is the body force, $\eta$ is the entropy, $q_{i}$ is the heat flux vector, $T_{0}$ is the uniform reference temperature, $h$ is the heat supply term, $\nu$ is the thermal displacement that satisfies that $\dot{\nu}=\theta$ is the temperature, $C_{i j k l}$ is the elasticity tensor that satisfies the major symmetry $C_{i j k l}=C_{k l i j}, \beta_{i j}$ is related with the tensor of thermal dilatation, $c$ is the heat capacity, $k_{i j}$ is the thermal conductivity tensor and satisfies the symmetry $k_{i j}=k_{j i}$ and $\kappa_{i j}$ is conductivity rate tensor which is typical for the theories of Green and Naghdi. It is worth recalling that this last tensor also satisfies the symmetry $\kappa_{i j}=\kappa_{j i}$.

If we substitute the constitutive equations into the evolution equations we obtain the field equations:

$$
\begin{gathered}
\rho \ddot{u}_{i}=\left(C_{i j k l} u_{k, l}-\beta_{i j} \theta\right)_{, j}+f_{i}, \\
c \ddot{\nu}-\left(\frac{\tau^{2}}{2} \kappa_{i j}^{*} \ddot{\nu}_{, j}\right)_{, i}=-\beta_{i j} \dot{u}_{i, j}+\left(\kappa_{i j}^{*} \nu_{, j}+\left(k_{i j}^{*}+\tau \kappa_{i j}^{*}\right) \dot{\nu}_{, j}\right)_{, i}+h .
\end{gathered}
$$

Here $k_{i j}^{*}=T_{0}^{-1} k_{i j}$ and $\kappa_{i j}^{*}=T_{0}^{-1} \kappa_{i j}$, however from now on we shall omit the star and we only write $k_{i j}$ and $\kappa_{i j}$ respectively.

To determine a well posed problem we should include some initial and boundary conditions. We assume the initial conditions

$u_{i}(x, 0)=u_{i}^{0}(x), \quad \dot{u}_{i}(x, 0)=v_{i}^{0}(x), \quad \nu(x, 0)=\nu^{0}(x), \quad \dot{\nu}(x, 0)=\theta^{0}(x), \quad x \in B$.

One possible family of homogeneous boundary conditions is:

$$
u_{i}(x, t)=\nu(x, 0)=0, \quad x \in \partial B, t>0 .
$$

\section{$3 \quad$ Uniqueness and instability}

In this section we consider the problem determined by the system (2.6), (2.7) the initial conditions (2.8) and the boundary conditions (2.9) and we use the logarithmic convexity argument to prove the uniqueness of solutions. We also prove the instability of solutions for the homogeneous version of this problem whenever the initial energy of the solution is negative.

The assumptions on the constitutive coefficients are the following: 
- The mass density $\rho$ and the heat capacity $c$ are strictly positive constants.

- The relaxation parameter $\tau$ is positive.

- The thermal conductivity tensor and the conductivity rate tensor are positive definite in the sense that there exist two positive constants $k_{0}$ and $\kappa_{0}$ such that

$$
k_{i j} \xi_{i} \xi_{j} \geq k_{0} \xi_{i} \xi_{i} \text {, and } \kappa_{i j} \xi_{i} \xi_{j} \geq \kappa_{0} \xi_{i} \xi_{i} \text {, for every vector } \xi_{i} \text {. }
$$

To prove a uniqueness theorem it is sufficient to prove that the only solution for the homogeneous version of the problem is the null solution. Therefore, in the remains of this section we will treat with the homogeneous version of the system $(2.6),(2.7)$.

We will start by considering the energy equation. We note that the energy

$$
\begin{gathered}
E(t)=\int_{B}\left(\rho \dot{u}_{i} \dot{u}_{i}+C_{i j k l} u_{i, j} u_{k, l}+c \theta^{2}+\frac{\tau^{2}}{2} \kappa_{i j} \theta_{, i} \theta_{, j}+\kappa_{i j} \nu_{, i} \nu_{, j}\right) d v \\
+2 \int_{0}^{t} \int_{B}\left(k_{i j}+\tau \kappa_{i j}\right) \theta_{, i}(s) \theta_{, j}(s) d v d s=E(0),
\end{gathered}
$$

is conserved.

The logarithmic convexity argument is based in the choice of a suitable function which satisfies some specific requirements. We consider several relations to define this function. First we integrate with respect to the time the homogeneous version of equation (2.7). We have

$$
\begin{gathered}
\int_{0}^{t}\left(\kappa_{i j} \nu_{, i}(s)\right)_{, j} d s+\left(\left(k_{i j}+\tau \kappa_{i j}\right) \nu_{, i}\right)_{, j}-\beta_{i j} u_{i, j}=c \theta-\frac{\tau^{2}}{2}\left(\kappa_{i j} \theta_{, i}\right)_{, j} \\
+\left(\left(k_{i j}+\tau \kappa_{i j}\right) \nu_{, i}^{0}\right)_{, j}-\beta_{i j} u_{i, j}^{0}-c \theta^{0}+\frac{\tau^{2}}{2}\left(\kappa_{i j} \theta_{, i}^{0}\right)_{, j}
\end{gathered}
$$

Now, we define $Q(x)$ as the solution to the Poisson equation

$$
\left(\kappa_{i j} Q_{, i}\right)_{, j}=c \theta^{0}-\frac{\tau^{2}}{2}\left(\kappa_{i j} \theta_{, i}^{0}\right)_{, j}+\beta_{i j} u_{i, j}^{0}-\left(\left(k_{i j}+\tau \kappa_{i j}\right) \nu_{, i}^{0}\right)_{, j},
$$

subject to the condition $Q=0$ at the boundary $\partial B$. If we denote

$$
\beta=\psi+Q, \text { where } \psi=\int_{0}^{t} \nu(s) d s,
$$

we obtain that equation (3.3) can be written as

$$
\left(\kappa_{i j} \beta_{, i}\right)_{, j}+\left(\left(k_{i j}+\tau \kappa_{i j}\right) \nu_{, i}\right)_{, j}-\beta_{i j} u_{i, j}=c \ddot{\beta}-\frac{\tau^{2}}{2}\left(\kappa_{i j} \ddot{\beta}_{, i}\right)_{, j} .
$$


We now define the function

$$
F_{\omega, t_{1}}(t)=\int_{B}\left(\rho u_{i} u_{i}+\kappa_{i j} \beta_{, i} \beta_{, j}+\int_{0}^{t}\left(k_{i j}+\tau \kappa_{i j}\right) \nu_{, i} \nu_{, j} d s\right) d v+\omega\left(t+t_{1}\right)^{2},
$$

where $\omega$ and $t_{1}$ are two constants to select later. We compute the two firsts derivatives of $F_{\omega, t_{1}}(t)$ :

$$
\begin{gathered}
\dot{F}_{\omega, t_{1}}=2 \int_{B}\left(\rho u_{i} \dot{u}_{i}+\kappa_{i j} \beta_{, i} \dot{\beta}_{, j}\right) d v+\int_{B}\left(k_{i j}+\tau \kappa_{i j}\right) \nu_{, i} \nu_{, j} d v+2 \omega\left(t+t_{1}\right) . \\
\ddot{F}_{\omega, t_{1}}=2 \int_{B}\left(\rho \dot{u}_{i} \dot{u}_{i}+\rho u_{i} \ddot{u}_{i}+\kappa_{i j} \dot{\beta}_{, i} \dot{\beta}_{, j}+\kappa_{i j} \beta_{, i} \ddot{\beta}_{, j}\right) d v+2 \int_{B}\left(k_{i j}+\tau \kappa_{i j}\right) \nu_{, i} \theta_{, j} d v+2 \omega .
\end{gathered}
$$

If we substitute into equations (2.6) and (3.6) and apply the divergence theorem we obtain that

$$
\ddot{F}_{\omega, t_{1}}=2 \int_{B}\left(\rho \dot{u}_{i} \dot{u}_{i}-C_{i j k l} u_{i, j} u_{k, l}+\kappa_{i j} \nu_{, i} \nu_{, j}-c \theta^{2}-\frac{\tau^{2}}{2} \kappa_{i j} \theta_{, i} \theta_{, j}\right) d v+2 \omega
$$

In view of the energy equation (3.2), we obtain that

$$
\ddot{F}_{\omega, t_{1}}=4 \int_{B}\left(\rho \dot{u}_{i} \dot{u}_{i}+\kappa_{i j} \nu_{, i} \nu_{, j}+\int_{0}^{t}\left(k_{i j}+\tau \kappa_{i j}\right) \theta_{, i} \theta_{, j} d s\right) d v+2(\omega-E(0)) .
$$

It is also worth noting that (3.8) can also be written as

$$
\begin{gathered}
\dot{F}_{\omega, t_{1}}=2 \int_{B}\left(\rho u_{i} \dot{u}_{i}+\kappa_{i j} \beta_{, i} \dot{\beta}_{, j}\right) d v+2 \int_{0}^{t} \int_{B}\left(k_{i j}+\tau \kappa_{i j}\right) \nu_{, i} \theta_{, j} d v d s \\
+\int_{B}\left(k_{i j}+\tau \kappa_{i j}\right) \nu_{, i}^{0} \nu_{, j}^{0} d v+2 \omega\left(t+t_{1}\right) .
\end{gathered}
$$

From (3.7), (3.12) and (3.11) we obtain that

$$
F_{\omega, t_{1}} \ddot{F}_{\omega, t_{1}}-\left(\dot{F}_{\omega, t_{1}}-\frac{\Gamma}{2}\right)^{2} \geq-2(E(0)+\omega) F_{\omega, t_{1}},
$$

where

$$
\Gamma=2 \int_{B}\left(k_{i j}+\tau \kappa_{i j}\right) \nu_{, i}^{0} \nu_{, j}^{0} d v .
$$

Inequality (3.13) is fundamental in our analysis. In case that we assume that the initial conditions vanish we have that $E(0)=0$ and $\Gamma=0$. If we take $\omega=t_{1}=0$ the inequality (3.13) becomes

$$
F \ddot{F}-(\dot{F})^{2} \geq 0,
$$

where we have simplified the notation and we use $F(t)$ for the function $F_{0,0}(t)$. From (3.15) we obtain the estimate

$$
F(t) \leq F(0)^{1-\frac{t}{T}} F(T)^{\frac{t}{T}}, \quad 0 \leq t \leq T .
$$


We then conclude that $F(t)$ vanishes for $0 \leq t \leq T$ and we get a uniqueness result.

In the general case from (3.13) we obtain that

$$
F_{\omega, t_{1}} \ddot{F}_{\omega, t_{1}}-\left(\dot{F}_{\omega, t_{1}}\right)^{2} \geq-\Gamma \dot{F}_{\omega, t_{1}},
$$

where we have selected $\omega=-E(0)$. It implies that

$$
\frac{d}{d t}\left(\frac{\dot{F}_{\omega, t_{1}}}{F_{\omega, t_{1}}}\right) \geq-\Gamma \frac{\dot{F}_{\omega, t_{1}}}{F_{\omega, t_{1}}^{2}}
$$

Therefore, we see that the function

$$
\frac{\dot{F}_{\omega, t_{1}}-\Gamma}{F_{\omega, t_{1}}}
$$

is increasing on time. In particular we see that

$$
\frac{\dot{F}_{\omega, t_{1}}(t)-\Gamma}{F_{\omega, t_{1}}(t)} \geq \frac{\dot{F}_{\omega, t_{1}}(0)-\Gamma}{F_{\omega, t_{1}}(0)} .
$$

Now, we select the arbitrary positive constant $t_{1}$ to be large enough to satisfy $\dot{F}_{\omega, t_{1}}(0)-\Gamma>0$. After a quadrature we obtain

$$
F_{\omega, t_{1}}(t) \geq \frac{F_{\omega, t_{1}}(0) \dot{F}_{\omega, t_{1}}(0)}{\dot{F}_{\omega, t_{1}}(0)-\Gamma} \exp \left(\frac{\dot{F}_{\omega, t_{1}}(0)-\Gamma}{F_{\omega, t_{1}}(0)}\right) t-\frac{\Gamma F_{\omega, t_{1}}(0)}{\dot{F}_{\omega, t_{1}}(0)-\Gamma} .
$$

Thus, we have established that $F(t)$ grows exponentially for large time.

We have proved:

Theorem 1 Under the conditions proposed in the begining of the section, we have:

- The first boundary value problem has at most a solution.

- If $E(0)<0$, then the solutions becomes unbounded in an exponential way.

It is worth noting that our uniqueness theorem applies to a large class of problems to the one proposed at [10]. In fact the new aspect is that we here do not assume that the elasticity tensor is positive definite. It is worth recalling that the logarithmic convexity argument is a powerful tool to study structural stability of thermoelastic systems. A such study could be proposed here after an easy modification of the function $F_{\omega, t_{1}}(t)$ that we have defined. However, we do not develop this analysis here and we only point out this possibility.

\section{Existence of solutions}

In this section we prove an existence theorem for the solutions of the thermoelastic problem proposed previously. However, in order to make the calculations 
easier, we consider the homogeneous one dimensional case. In particular all the constitutive tensors become constants.

We assume that the body occupies the interval $[0, \pi]$. That is the system of field equations becomes

$$
\begin{gathered}
\rho \ddot{u}=\mu u_{x x}-\beta \dot{\nu}_{x}, \\
c \ddot{\nu}-\frac{\tau^{2} \kappa}{2} \ddot{\nu}_{x x}=\kappa \nu_{x x}+(k+\tau \kappa) \dot{\nu}_{x x}-\beta \dot{u}_{x} .
\end{gathered}
$$

Although Dirichlet boundary conditions could be considered, in this case the analysis would be easier if we consider the boundary conditions

$$
u(x, t)=\nu_{x}(x, t)=0, \quad x=0, \pi, t \geq 0 .
$$

We should impose that

$$
\int_{0}^{\pi} \nu^{0} d x=\int_{0}^{\pi} \theta^{0} d x=0
$$

in order to save constant solutions.

In the remains of this paper we assume that

- $\rho, \mu, c, k, \kappa$ and $\tau$ are positive constants.

If we denote $\dot{u}=v, \dot{\nu}=\theta$ and $D=d / d x$, we can write the system in the following form:

$$
\begin{gathered}
\dot{u}=v \\
\dot{v}=\frac{1}{\rho}\left(\mu D^{2} u-\beta D \theta\right) \\
\dot{\nu}=\theta \\
\dot{\theta}=\left(c I-\frac{\tau^{2} \kappa}{2} D^{2}\right)^{-1}\left(\kappa D^{2} \nu+(k+\tau \kappa) D^{2} \theta-\beta D v\right) .
\end{gathered}
$$

We define $L_{*}^{2}=\left\{f \in L^{2} ; \int_{0}^{\pi} f d x=0\right\}$ and let $H_{*}^{1}=H^{1} \cap L_{*}^{2}$. To formalize the problem, we assume the evolution to be taking place on the Hilbert space

$$
\mathcal{H}=\left\{U=(u, v, \nu, \theta) ; u \in H_{0}^{1}, v \in L^{2}, \nu \in H_{*}^{1}, \theta \in H_{*}^{1}\right\},
$$

driven by the operator

$$
\mathcal{A}=\left(\begin{array}{cccc}
0 & I & 0 & 0 \\
\frac{\mu}{\rho} D^{2} & 0 & 0 & -\frac{\beta}{\rho} D \\
0 & 0 & 0 & I \\
0 & \left(c I-\frac{\tau^{2} \kappa}{2} D^{2}\right)^{-1} \beta D & \left(c I-\frac{\tau^{2} \kappa}{2} D^{2}\right)^{-1} \kappa D^{2} & \left(c I-\frac{\tau^{2} \kappa}{2} D^{2}\right)^{-1}(k+\tau \kappa) D^{2}
\end{array}\right)
$$

With the aforementioned notation, our initial boundary value problem can be written in abstract form as

$$
\frac{d U}{d t}=\mathcal{A} U, \quad U^{0}=\left(u^{0}, v^{0}, \nu^{0}, \theta^{0}\right) .
$$


It can be proved that the mild solutions of system are given by the semigroup of contractions generated by the operator $\mathcal{A}$. We define an inner product in $\mathcal{H}$. If $U^{*}=\left(u^{*}, v^{*}, \nu^{*}, \theta^{*}\right)$, then

$$
<U, U^{*}>_{\mathcal{H}}=\frac{1}{2} \int_{0}^{\pi}\left(\rho v \bar{v}^{*}+c \theta \bar{\theta}^{*}+\frac{\tau^{2} \kappa}{2} \theta_{x} \bar{\theta}_{x}^{*}+\mu u_{x} \bar{u}_{x}^{*}+\kappa \nu_{x} \bar{\nu}_{x}^{*}\right) d x .
$$

Here, a superposed bar denotes the complex conjugation. It should be pointed out that this product is equivalent to the usual product in the Hilbert space $\mathcal{H}$. It is worth noting that the squared norm of a vector is given by

$$
\left.|| U\right|_{\mathcal{H}}=\frac{1}{2} \int_{0}^{\pi}\left(\rho|v|^{2}+c|\theta|^{2}+\frac{\tau^{2} \kappa}{2}\left|\theta_{x}\right|^{2}+\mu\left|u_{x}\right|^{2}+\kappa\left|\nu_{x}\right|^{2}\right) d x .
$$

The domain $\mathcal{D}(\mathcal{A})$ is the set of $U \in \mathcal{H}$ such that $\mathcal{A} U \in \mathcal{H}$.

Lemma 1 The operator $\mathcal{A}$ defined previously is the infinitesimal generator of a $C_{0}$-semigroup of contractions on $\mathcal{H}$.

PROOF. First of all, we notice that $\mathcal{D}(\mathcal{A})$ contains a subset that is dense in $\mathcal{H}$ and then $\mathcal{D}(\mathcal{A})$ is also dense in $\mathcal{H}$. We will show that $\mathcal{A}$ is a dissipative operator and that 0 is in the resolvent set of $\mathcal{A}$. Using the Lumer-Phillips theorem, the conclusion will follow.

On the one side, a direct calculation gives

$$
\Re<\mathcal{A} U, U>_{\mathcal{H}}=-\frac{1}{2} \int_{0}^{\pi}(k+\tau \kappa)\left|\theta_{x}\right|^{2} d x .
$$

and, therefore, the operator $\mathcal{A}$ is dissipative. On the other hand, for any $F=$ $\left(f_{1}, f_{2}, f_{3}, f_{4}\right) \in \mathcal{H}$ we will find a unique $U \in \mathcal{D}(\mathcal{A})$ such that $\mathcal{A} U=F$, or equivalently

$$
\begin{gathered}
v=f_{1}, \\
\mu D^{2} u-\beta D \theta=\rho f_{2}, \\
\theta=f_{3}, \\
\kappa D^{2} \nu+(k+\tau \kappa) D^{2} \theta-\beta D v=c f_{4}-\frac{\tau^{2} \kappa}{2} D^{2} f_{4} .
\end{gathered}
$$

The second and fourth equations can be written in terms of $f_{1}, f_{2}, f_{3}$ and $f_{4}$ as follows:

$$
\begin{gathered}
\mu D^{2} u=\rho f_{2}+\beta D f_{3}, \\
\kappa D^{2} \nu=c f_{4}-\frac{\tau^{2} \kappa}{2} D^{2} f_{4}+\beta D f_{1}-(k+\tau \kappa) D^{2} f_{3}
\end{gathered}
$$

To prove the solvability of this system we develop $f_{1}, \ldots, f_{4}$ in Fourier series. The families of $\sin (n x)$ and $\cos (m x)$ are two orthonormal complete system in the Hilbert space $L^{2}$. In particular, we develop $f_{1}$ and $f_{2}$ in series of sines and $f_{3}$ and $f_{4}$ in series of cosines. So, we take $f_{1}=\sum_{n=1}^{\infty} a_{n} \sin (n x), f_{2}=\sum_{n=1}^{\infty} b_{n} \sin (n x)$, $f_{3}=\sum_{n=1}^{\infty} c_{n} \cos (n x)$ and $f_{4}=\sum_{n=1}^{\infty} d_{n} \cos (n x)$. In view of the fact that the 
averages of $f_{3}$ and $f_{4}$ are zero, the developments in the cosines series start from $n=1$.

We will show that it is possible to find $u=\sum_{n=1}^{\infty} u_{n} \sin (n x)$ and $\nu=$ $\sum_{n=1}^{\infty} \nu_{n} \cos (n x)$ such that $\sum_{n=1}^{\infty} n^{2}\left|u_{n}\right|^{2}<\infty$ and $\sum_{n=1}^{\infty} n^{2}\left|\nu_{n}\right|^{2}<\infty$. We can take

$$
u_{n}=\frac{\beta n c_{n}-\rho b_{n}}{\mu n^{2}}
$$

and

$$
\nu_{n}=-\frac{c d_{n}+\frac{\tau^{2} \kappa}{2} n^{2} d_{n}+\beta n a_{n}+(k+\tau \kappa) n^{2} c_{n}}{\kappa n^{2}} .
$$

Thus, it is clear that $u$ and $\nu$ satisfy the desired conditions. It is not difficult to see that $\|U\| \leq C\|F\|$. Therefore, 0 is in the resolvent set of $\mathcal{A}$ and the lemma is proved.

Theorem 2 The problem given by system (6.1), (6.2) with boundary conditions (4.3) and initial conditions (2.8) in $\mathcal{H}$ has a unique mild solution.

PROOF. The proof is a direct consequence of the previous lemma.

It is worth noting that the semigroup is dissipative. The stability of the system is a consequence of this fact.

\section{Slow decay}

The aim of this section is to prove that the decay of the solutions obtained in the previous section is slow. We mean that there is no exponential decay of solutions. To this end we are going to study the point spectrum of the solutions and we will see that there always exist solutions as near as we want to the imaginary axis.

The solution in this case will be combinations of functions of the form

$$
u=A \exp (\omega t) \sin n x, \quad \nu=B \exp (\omega t) \cos n x .
$$

Imposing them as solutions of the system we obtain the following homogeneous system with the unknowns $A, B$ :

$$
B n \beta \omega-A\left(n^{2} \mu+\rho \omega^{2}\right)=0, \quad 2 A n \beta \omega+B\left(2 c \omega^{2}+n^{2}(2 k \omega+\kappa(2+\tau \omega(2+\tau \omega))=0\right.
$$

We want to obtain a nontrivial solution. We impose that the determinant of this system vanishes. Therefore $\omega$ must satisfy the equation

$$
\begin{gathered}
2 n^{4} \kappa \mu+2 x\left(k n^{4} \mu+n^{4} \kappa \mu \tau\right)+2 x^{3}\left(k n^{2} \rho+n^{2} \kappa \rho \tau\right) \\
+x^{2}\left(2 n^{2} \beta^{2}+2 c n^{2} \mu+2 n^{2} \kappa \rho+n^{4} \kappa \mu \tau^{2}\right)+x^{4}\left(2 c \rho+n^{2} \kappa \rho \tau^{2}\right)=0
\end{gathered}
$$

Our aim is to prove that solutions to this equation as near as we want to the complex axis can be found. Therefore, it will be sufficient to show that for $\epsilon$ as small as we want, we can find solutions which are on the right of the line 
$\Re\{z\}=-\epsilon$. This is equivalent to prove that there exists a solution of the equation

$$
\begin{gathered}
2 n^{4} \kappa \mu+2(x-\epsilon)\left(k n^{4} \mu+n^{4} \kappa \mu \tau\right)+2(x-\epsilon)^{3}\left(k n^{2} \rho+n^{2} \kappa \rho \tau\right) \\
+(x-\epsilon)^{2}\left(2 n^{2} \beta^{2}+2 c n^{2} \mu+2 n^{2} \kappa \rho+n^{4} \kappa \mu \tau^{2}\right)+(x-\epsilon)^{4}\left(2 c \rho+n^{2} \kappa \rho \tau^{2}\right)=0,
\end{gathered}
$$

with positive real part.

We prove it by means of the Hurwitz theorem that says that the necessary and sufficient condition to guarantee that the solutions of the equation

$$
l_{4} x^{4}+l_{3} x^{3}+l_{2} x^{2}+l_{1} x+l_{0}=0,
$$

have negative real part is that $l_{i}>0, l_{3} l_{2}>l_{4} l_{1}$ and $l_{1} l_{2} l_{3}-l_{0} l_{3}^{2}-l_{4} l_{1}^{2}>0$.

In our case, we can write equation (5.4) as

$$
\begin{aligned}
\rho\left(2 c+n^{2} \kappa \rho \tau^{2}\right) x^{4}+ & \left(2 \rho(k+\kappa \tau+) n^{2}+P_{3}(\epsilon)\right) x^{3}+\left(2\left(\beta^{2}+c \mu+\kappa \rho\right) n^{2}+\kappa \mu \tau^{2} n^{4}+P_{2}(\epsilon)\right) x^{2} \\
& +2\left((k \mu+\kappa \mu \tau) n^{4}+P_{1}(\epsilon)\right) x+2 \kappa \mu n^{4}+P_{0}(\epsilon)
\end{aligned}
$$

where

$$
\begin{gathered}
P_{3}=-4 \epsilon \rho\left(\kappa \tau^{2} n^{2}+2 c\right), \quad P_{2}=6 \epsilon\left(-k \rho-\kappa \rho \tau+\epsilon \rho \kappa \tau^{2}\right) n^{2}+6 c \rho \epsilon^{2} \\
P_{1}=\epsilon\left(-\kappa \mu \tau^{2} n^{4}-\left(2 \beta^{2}+2 c \mu+2 \kappa \rho-3 k \rho \epsilon-3 \kappa \rho \tau \epsilon+2 \kappa \rho \tau^{2} \epsilon^{2}\right) n^{2}-4 c \rho \epsilon^{2}\right) \\
P_{0}=2 c \epsilon^{4} \rho-n^{4}\left(2 k \epsilon \mu+2 \epsilon \kappa \mu \tau-\epsilon^{2} \kappa \mu \tau^{2}\right) \\
-n^{2}\left(-2 \beta^{2} \epsilon^{2}-2 c \epsilon^{2} \mu+2 k \epsilon^{3} \rho-2 \epsilon^{2} \kappa \rho+2 \epsilon^{3} \kappa \rho \tau-\epsilon^{4} \kappa \rho \tau^{2}\right) .
\end{gathered}
$$

Direct calculations allow us to see that there exists $n$ large enough to guarantee that $l_{3} l_{2}<l_{4} l_{1}$ and $l_{1} l_{2} l_{3}-l_{0} l_{3}^{2}-l_{4} l_{1}^{2}<0$. We have that

$$
\begin{gathered}
l_{3} l_{2}-l_{4} l_{1}=-2 \epsilon \kappa^{2} \mu \rho \tau^{4} n^{6}+\left(4 k \kappa \rho^{2}+4 \kappa^{2} \rho^{2} \tau+4 k \beta^{2} \rho+4 \beta^{2} \kappa \rho \tau-20 \epsilon^{3} \kappa^{2} \rho^{2} \tau^{4}+\right. \\
\left.-\epsilon\left(12 k^{2} \rho^{2}+24 k \kappa \rho^{2} \tau+8 c \kappa \mu \rho \tau^{2}+16 \kappa^{2} \rho^{2} \tau^{2}+4 \beta^{2} \kappa \rho \tau^{2}\right)+\epsilon^{2}\left(30 k \kappa \rho^{2} \tau^{2}+30 \kappa^{2} \rho^{2} \tau^{3}\right)\right) n^{4} \\
+n^{2}\left(-\epsilon\left(8 c \beta^{2} \rho+8 c^{2} \mu \rho+8 c \kappa \rho^{2}\right)-80 c \epsilon^{3} \kappa \rho^{2} \tau^{2}+\epsilon^{2}\left(60 c k \rho^{2}+60 c \kappa \rho^{2} \tau\right)\right) \\
-80 c^{2} \epsilon^{3} \rho^{2} .
\end{gathered}
$$

It is clear that for $n$ large enough this expression becomes negative because the coefficient of the larger degree in the variable $n$ is negative. Therefore this expression is negative and the decay is slow.

It is worth noting that an analysis similar could be done for the expression $l_{1} l_{2} l_{3}-l_{0} l_{3}^{2}-l_{4} l_{1}^{2}$, but we do not reproduce it here to save cumbersome calculations. Nevertheless it is sufficient with the analysis of $l_{3} l_{2}-l_{4} l_{1}$ to prove the slow decay. 


\section{Further comments}

If $\tau_{\nu}<\tau_{q}$ we have that $\tau<0$. Therefore our system becomes

$$
\begin{gathered}
\rho \ddot{u}=\mu u_{x x}-\beta \dot{\nu}_{x}, \\
c \ddot{\nu}-\frac{\left(\tau^{*}\right)^{2} \kappa}{2} \ddot{\nu}_{x x}=\kappa \nu_{x x}+\left(k-\tau^{*} \kappa\right) \dot{\nu}_{x x}-\beta \dot{u}_{x},
\end{gathered}
$$

where $\tau^{*}=\tau_{q}-\tau_{\nu}$. An existence theorem can be obtained for this case following an argument similar to the one developed in Section 4.

In case that $k>\tau^{*} \kappa$ we can see that the system is stable. However when $k<\tau^{*} \kappa$ the system is not stable. In fact, we can look for solutions of the form (5.1). If we look for nontrivial solutions, then $\omega$ must satisfy the equation

$$
\begin{gathered}
2 n^{4} \kappa \mu+2 x\left(n^{2} \beta^{2}+k n^{4} \mu-n^{4} \kappa \mu \tau\right)+2 x^{3}\left(k n^{2} \rho-n^{2} \kappa \rho \tau\right) \\
+x^{2}\left(2 c n^{2} \mu+2 n^{2} \kappa \rho+n^{4} \kappa \mu \tau^{2}+2 n^{2} \beta^{2}\right)+x^{4}\left(2 c \rho+n^{2} \kappa \rho \tau^{2}\right)=0,
\end{gathered}
$$

where we have omitted the star to make the written easier.

This is an equation of the type (5.5). For $n$ large enough the coefficient $2\left(n^{2} \beta^{2}+k n^{4} \mu-n^{4} \kappa \mu \tau\right)$ becomes negative. Alternatively if we have that

$$
l_{3} l_{2}-l_{4} l_{1}=4 n^{4} \rho\left(\beta^{2}+\kappa \rho\right)(k-\kappa \tau)
$$

which is also negative and then we see the instability of solutions.

\section{References}

[1] K. Borgmeyer, R. Quintanilla and R. Racke, Phase-lag heat conduction: decay rates for limit problems and well-posedness, J. Evolution Equations 14 (2014), 863-884.

[2] S. K. R. Choudhuri, On a thermoelastic three-phase-lag model, J. Thermal Stresses, 30(2007), 231-238.

[3] M. Dreher, R. Quintanilla and R. Racke, Ill-posed problems in thermomechanics, Appl. Math. Letters 22(2009), 1374-1379.

[4] A. E. Green and K. A. Lindsay, Thermoelasticity, J. Elasticity, 2(1972), 1-7.

[5] A. E. Green and P. M. Naghdi, On undamped heat waves in an elastic solid, J. Thermal Stresses 15(1992), 253-264.

[6] A. E. Green and P. M. Naghdi, Thermoelasticity without energy dissipation, J. Elasticity, 31(1993), 189-208.

[7] M. C. Leseduarte and R. Quintanilla, Phragmen-Lindelof alternative for an exact heat conduction equation with delay, Communications Pure Appli. Analysis 12(2013), 1221-1235. 
[8] Z. Liu and S. Zheng, Semigroups Associated with Dissipative Systems, Chapman \& Hall/CRC Boca Raton (1999).

[9] H. W. Lord and Y. Shulman, A generalized dynamical theory of thermoelasticity, Jour. Mech. Phys. Solids, 15(1967), 299-309.

[10] B. Kumari and S. Mukhopadhayay, Some theorems on linear theory of thermoelasticity for an anisotropic medium under an exact heat conduction model with a delay. Mathematics and Mechanics of Solids, (2016). doi:10.1177/1081286515620263.

[11] A. Magaña and R. Quintanilla, Uniqueness and growth of solutions in twotemperature generalized thermoelastic theories, Mathematics and Mechanics of Solids, 14(2009), 622-634.

[12] A. Miranville and R. Quintanilla, A phase-field model based on a threephase-lag heat conduction, Applied Mathematics and Optimization, 63(2011), $133-150$.

[13] S. Mukhopadhyay, S. Kothari and R. Kumar. On the representation of solutions for the theory of generalized thermoelasticity with three phase-lags, Acta Mechanica, 214(2010), 305-314.

[14] R. Quintanilla, Structural stability and continuous dependence of solutions of thermoelasticity of type III, Discrete and continuous Dynamical Systems B, 1(2001), 463-470.

[15] R. Quintanilla, Exponential stability and uniqueness in thermoelasticity with two temperatures. Dynamics of Continuous, Discrete and Impulsive Systems A, 11(2004), 57-68.

[16] R. Quintanilla, A well-posed problem for the dual-phase-lag heat conduction. Journal of Thermal Stresses, 31(2008), 260-269.

[17] R. Quintanilla, A well-posed problem for the three-dual-phase-lag heat conduction. Journal of Thermal Stresses, 32(2009), 1270-1278.

[18] R. Quintanilla, Some solutions for a family of exact phase-lag heat conduction problems, Mechanics Research Communications, 38(2011), 355-360.

[19] R. Quintanilla and R. Racke, Stability in thermoelasticity of type III, Discrete and continuous Dynamical Systems B, 3(2003), 383-400.

[20] R. Quintanilla and R. Racke, A note on stability of dual-phase-lag heat conduction, Int. J. Heat Mass Transfer, 49(2006), 1209-1213.

[21] R. Quintanilla and R. Racke, Qualitative aspects in dual-phase-lag heat conduction, Proc. Royal Society London A., 463(2007), 659-674.

[22] R. Quintanilla and R. Racke, A note on stability in three-phase-lag heat conduction, Int. J. Heat Mass Transfer, 51(2008), 24-29. 
[23] R. Racke, Thermoelasticity with second sound exponential stability in linear and non-linear 1-d, Mathematical Methods Applied Sciences, 25(2002) 409-441.

[24] D. Y. Tzou, A unified approach for heat conduction from macro to microscales, ASME J. Heat Transfer, 117(1995), 8-16. 